Fecha de recepción: julio 2021

Fecha de aprobación: agosto 2021

Fecha publicación: septiembre 2021

\section{La intersubjetividad como estrategia de comunicación para la reactivación de las actividades deportivas sociales luego de la pandemia}

Kléver Rolando Samaniego Pesantez ${ }^{(1)}$ y Silvia

Lorena Álvarez León ${ }^{(2)}$

\begin{abstract}
Resumen: El mundo tiene puesto su mirada en la búsqueda de una vacuna para eliminar o neutralizar definitivamente al Corona Virus (Covid-19), declarado como pandemia en marzo de 2020. El confinamiento, el distanciamiento social y los juegos en dispositivos electrónicos se están constituyendo en un obstáculo para la reactivación de las actividades físicas en tiempos post-pandemia. Es por esa razón, que propongo iniciar con un pensamiento prospectivo para disminuir los efectos negativos que podrían afectar al momento de reactivar las actividades físicas. Con este objetivo, realizaremos una exploración comunicacional por los procesos intersubjetivos existentes en las Jornadas Deportivas Vacacionales de Sígsig-Ecuador, considerada la más constante y antigua del Ecuador, con la intensión de examinar las estrategias de comunicación utilizadas en su convocatoria y participación.
\end{abstract}

Palabras clave: Intersubjetividad - Comunicación - Enactiva - Jornadas Deportivas Vacacionales de Sígsig - Ecuador - Matrices Socioculturales.

[Resúmenes en inglés y portugués en las páginas 37-38]

(1) Silvia Lorena Álvarez León. Candidata a Doctora en Comunicación por la Universidad de la Habana - Cuba. Magister en Comunicación y Marketing por la Universidad del Azuay. Cuenca-Ecuador Licenciada en Comunicación Social por la Universidad del Azuay. Cuenca-Ecuador. 15 años de experiencia en el manejo de relaciones públicas en el sector privado con especialización en centros comerciales. Actualmente también es productora de contenidos y locutora de radio en señal abierta.Mail: shimuqui@hotmail.com

${ }^{(2)}$ Kléver Rolando Samaniego Pesantez. Doctor en comunicación por la Universidad de La Plata-Argentina, Magister en Gestión de la Comunicación Política y Electoral de la Universidad Autónoma de Barcelona-España y Lcdo. en Comunicación Social por la Universidad de Cuenca. Actualmente es parte del grupo de consultores estratégicos de Rosario - Argentina. Mail: cenitp@hotmail.com 


\section{Introducción}

Jornadas Deportivas Vacacionales (JDV) del cantón Sígsig es el evento deportivo más constante y antiguo de la República del Ecuador. Este acontecimiento se realiza año tras año desde 1972, el cual se desarrolla en una sola semana durante la temporada vacacional estudiantil. Dicho evento congrega a más de 10.000 visitantes, además cuenta con 1500 deportistas que sumados 5000 habitantes de la zona urbana, llegan alrededor de 16.000 personas en un solo lugar. La zona central de Sígsig no contempla las comodidades de alojamiento necesarias para los turistas y deportistas que asisten al evento.

$\mathrm{Al}$ frente de este acontecimiento social surgen las siguientes interrogantes: ¿Qué hace tan llamativo a las Jornadas Deportivas Vacacionales de Sígsig-Ecuador? ¿Por qué a medida que avanza el tiempo aumenta la presencia de turistas, jugadores y espectadores a pesar de las incomodidades que sufren? ¿Por qué el deporte logra congregar a diferentes públicos en espacios sociales? ¿Qué papel cumple la comunicación en ese proceso? ¿Es posible con procesos intersubjetivos propiciar la participación deportiva?

Para responder estas inquietudes analizaremos las estrategias, utilizadas por el Comité Organizador de las JDV de Sígsig, para la convocatoria y participación de deportistas, espectadores y turistas asistentes a mencionado evento deportivo. En ese contexto, exploraremos por la intersubjetividad como un proceso del encuentro sociocultural conversacional que enactúa (Massoni, 2003). Esto "implica la consideración de lo complejo y de lo fluido en tanto modalidad de la relación intersubjetiva que es propia de lo comunicacional" (ib. 2015, p. 3). Lo que permitirá considerar y reconocer qué sinergias, convergencias y estrategias enactúan en la relación Comité Rector, espectadores y deportistas donde el acto de participar conlleva vínculos intersubjetivos que se reconfiguran en la comunicación misma.

Este trabajo se realiza con la finalidad de constituirla en una alternativa para la reactivación de las actividades físicas post-pandemia, desde el Ecuador, y para toda "América Latina donde la exclusión (social, política y cultural) es la característica más fuerte y determinante" (Uranga, 2008, p. 4).

\section{Dispositivo investigativo}

La motivación de esta investigación surge con la necesidad de conocer y analizar determinadas formas de convocatoria en el espacio deportivo y cultural, realizadas por el Comité Rector de las Jornadas Deportivas Vacacionales en el periodo 1972-2019 desde la perspectiva de la Comunicación Estratégica Enactiva. Caracterizándolas y entendiéndolas como proceso intersubjetivo para la participación en eventos deportivos.

Es apropiado destacar que la literatura académica sobre la intersubjetividad ha surgido de forma relativamente independiente en el ámbito de la filosofía evolutiva y clínica, la psicología evolutiva -enfocada específicamente en la relación madre e hijo-.

La intersubjetividad como concepto tiene sus primeras pulsaciones en la filosofía cartesiana donde se visibiliza la intención de conocer la mente del otro, aduciendo que, la única 
mente de la cual se tiene conocimiento "directo", "infalible" e "incorregible" es la mente propia (Cely, 2014). No obstante, Benjamin (1990) y Natterson y Friedman (1995) señalan que también Hegel (1807), había descrito ciertos procesos importantes para comprender la intersubjetividad en su fenomenología del espíritu.

La filosofía cartesiana con el concepto de intersubjetividad ha buscado la variedad de posibles relaciones entre las distintas perspectivas de los individuos e implica la tendencia humana a reconocer y tener en cuenta la experiencia personal o subjetiva del otro (Gillespie \& Cornish, 2009; Neimeyer, 1998), también ha sido utilizada para referirse a la posibilidad de distintos observadores para compartir una definición u opinión de un objeto, o cierto estado de las cosas, atribuyéndose cierta objetividad y neutralidad a los observadores (Orange, 1997 en Sassenfeld, 2010; Mori \& Hayashi, 2006 en Gillespie \& Cornish, 2009). Otros autores como Laing, Phillipson y Lee (1966 en Gillespie \& Cornish, 2009) la han definido también en términos de una mutua conciencia de acuerdo o desacuerdo.

La concepción cartesiana de lo mental insiste en que, la discusión sobre el tema de las otras mentes ha oscilado siempre entre dos posiciones extremas -la solipsista y la conductistaporque se parte de algunas intuiciones equivocadas (Bruner, 2000). Además, afirma que los sentimientos y los pensamientos de los otros se manifiestan en sus expresiones y acciones. Bruner (2000) considera que la vida mental del otro es, en algún sentido, inaccesible. Cely (2014) manifiesta que es erróneo afirmar que la vida mental de los otros es esencialmente inaccesible, pero también parece erróneo afirmar que todo está a la vista. El reto es conciliar las dos intuiciones, más que abandonar una de ellas. El conocimiento de los otros depende de la interacción, concepto que, en este sentido, se opone a los privilegios tanto de la introspección como de la observación conductual.

La noción del concepto intersubjetividad se fortalece en la filosofía fenomenológica, especialmente en las contribuciones de Husserl y Merleau-Ponty (1950). Husserl (1950) ha propuesto una manera radicalmente anticartesiana de entender la propia mente y la mente de los otros. El autor hace énfasis en que la experiencia subjetiva no se debe concebir como un reino interno de estados mentales privados, separados del cuerpo y definidos solipsísticamente. Se propone entender que la subjetividad en los gestos y las posturas corporales, así como en el mismo comportamiento, es decir, se constituye en un contexto de interacción con otros; se habla así del surgimiento intersubjetivo de lo subjetivo.

La teoría de la subjetividad de la filosofía fenomenológica, es un movimiento filosófico del siglo XX, que describe las estructuras de la experiencia como tal y como se presenta en la conciencia sin recurrir a teorías, deducciones, o suposiciones procedentes de otras disciplinas tales como las ciencias naturales (García, 2010).

La fenomenología se basa en las vivencias y trata de examinar de forma sistemática los tipos y formas de experiencias internas de los sujetos, teniendo como fin último la comprensión de ser humano. Por tanto, una perspectiva más basada en la intuición y la vivencia que en el análisis y la explicación. Esta corriente de la filosofía considera que no se pueden comprender al hombre y al mundo si no es a partir de la facticidad, es decir, de los hechos. Es una filosofía para entender que el mundo está siempre "ahí", antes de la reflexión, como una presencia inalienable.

En estas afirmaciones se pone en evidencia la dualidad de la fenomenología: por un lado, pretende erigirse como una ciencia rigurosa; pero por la otra, también tiene como fin dar 
cuenta del espacio, del tiempo y del mundo vivido. Es el estudio de las esencias de las cosas y emociones.

El filósofo alemán Max Scheler en 1910, definió la fenomenología como el estudio de estructuras de la conciencia que capacitan al conocimiento para referirse a los objetos fuera de sí misma. Alfred Schütz (1972) realiza una interrogante básica: ¿dónde y cómo se forman los significados de la acción social? cuyo problema básico es la cuestión de la sociabilidad como forma superior de intersubjetividad.

Esta preocupación básica parte de varias ideas importantes: el estudio de la vida social no puede excluir al sujeto; este está implicado en la construcción de la realidad objetiva que estudia la ciencia social; el elemento central es, entonces, el fenómeno-sujeto. El énfasis no se encuentra ni en el sistema social ni en las relaciones funcionales que se dan en la vida en sociedad, sino en la interpretación de los significados del mundo (Lebenswelt) y las acciones e interacciones de los sujetos sociales. Del mundo conocido y de las experiencias intersubjetivas compartidas por los sujetos, se obtienen las señales, las indicaciones para interpretar la diversidad de símbolos (Berger, Peter; Luckmann, \& Thomas, 1993, p. 25).

Para Schütz (1993), la realidad es un mundo en el que los fenómenos están dados, sin importar si éstos son reales, ideales o imaginarios. Este mundo es el mundo de la vida cotidiana, los sujetos viven en una actitud natural, cuya materia prima es el sentido común. El sujeto realiza acciones que están cargadas de significados. Todas sus acciones tienen un sentido; aunque el actor no haya tenido intención de significar algo, su acción puede ser interpretada por otro. Las vivencias son interpretadas subjetivamente, pues el sujeto recurre a su repositorio de conocimiento disponible, para asociar aquello que se conoce a lo que se desconoce.

Para Schütz (1993), el "alter ego u otro yo" le es dado al sujeto como una demostración práctica de un ser idéntico con quien comparte un mundo intersubjetivo conocido como "mundo del Yo" en el cual conviven tanto sus antecesores, contemporáneos y predecesores. Esto significa que el "otro" es como "yo", capaz de actuar y de pensar; que su capacidad de pensamiento es igual a la mía en su totalidad; que análogamente a mi vida, la de él muestra la misma forma estructural-temporal con todas las experiencias que ello conlleva. Significa, conjuntamente, que el "otro", como "yo", puede proyectarse sobre sus actos y pensamientos, dirigidos hacia sus objetos, o bien volverse hacia su sí-mismo de modo pretérito, pero puede contemplar mi flujo de conciencia en un presente vívido; que, por lo tanto, tiene la legítima experiencia de envejecer conmigo y viceversa.

La propuesta de Schütz es la intersubjetividad, comprendida como fundamento de la vida social, como relación entre sujetos que provee de sentidos y significados a las acciones que cada uno de ellos realiza en el mundo de la vida cotidiana. La intersubjetividad, por tanto, es el escenario en el que se desarrolla toda relación de interacción. La interacción es siempre comunicación con otro distinto a uno mismo, y es mediante este proceso que los sujetos sociales adquieren capacidad reflexiva para verse a sí mismos y para instituir o dar forma y sentido a la realidad social que los rodea. La aparición de los otros es para el hombre un fenómeno complejo (Galindo, 2003). 
Las principales aportaciones de Alfred Schütz al pensamiento sobre lo social son, por un lado, la incorporación del mundo cotidiano a la investigación sociológica, a partir de la reivindicación como objeto de estudio de la sociología el ámbito de la sociabilidad, es decir, el conjunto de las relaciones interpersonales y de las actitudes de la gente que son pragmáticamente reproducidas o modificadas en la vida cotidiana; y por el otro, la definición propia de las características del mundo de la vida, a saber: Que sus significados son construcciones sociales; que es intersubjetivo; que está conformado por personas que viven en él con una actitud natural, es decir, que se toman las cosas de manera arcaica, irreflexiva y práctica, tal y como aparecen; que es un ámbito familiar en el que los sujetos se mueven con un acervo de conocimiento a mano (Pech, Rizo, \& Romeu, 2008).

Otro de los aportes importantes desde la neurofenomenología es el autor chileno Francisco Varela (2000) en su artículo el fenómenos de la vida presenta cuatro pautas para mirar el futuro de las ciencias cognitivas y menciona como tercer punto la intersubjetividad como el poder constitutivo de la empatía y enfatiza que la ciencia cognitiva muestran claramente que la individualidad y la intersubjetividad no se oponen, sino que son necesariamente complementarias, que son también emocional-afectivos. El autor dice que se debe mirar al Otro, no como una cosa, sino como otra subjetividad semejante a la mía, un alter ego. Es a través del cuerpo del otro que establezco un vínculo con el otro, primero como organismo semejante al mío, pero también percibido como presencia encarnada, lugar y medio de un campo experiencial y presenta a la empatía, como el camino real para acceder a la vida social consciente; más allá de la simple interacción, como inter-subjetividad fundamental.

El conocimiento de sentido común es el conocimiento del mundo de la vida. Este conocimiento, por tanto, no es privado, sino intersubjetivo y socializado, compartido por los sujetos que en ese mundo interactúan, se vinculan. Es por medio de la comunicación que el sujeto transmite sus conocimientos a sus semejantes, y de alguna manera transmite estos conocimientos a sus predecesores. Por ello, la comunicación es considerada como el medio tipificado o significador por excelencia (Rizo, 2012). En los últimos años la intersubjetividad ha sido abordada por la comunicación desde dos tendencias claramente marcadas, la primera subyace desde la sociología, con la Asociación Mexicana de Investigadores de la Comunicación (AMIC) representada en Antonio Pascuali (2007) y la segunda se desprende de investigadores de América del Sur con base en la Teoría de Comunicación Estratégica Enactiva de la Universidad de Rosario de Sandra Massoni (2003).

La comunicación intersubjetiva es la línea de investigación que nace de la propuesta de Antonio Pascuali en el XIX Encuentro de la Asociación Mexicana de Investigadores de la Comunicación (AMIC) desarrollado en el año 2007. Esta teoría está siendo trabajada en base a dos vertientes: entre los investigadores con enfoques sociológicos encontramos a George Herbert Mead $(1938,1986)$ y Erving Goffman (1938) Alfred Schutz (1974), Richard Sennett (2009), Randall Collins (2009) entre otros, y en los enfoques filosóficos a Martin Heidegger (2006), Jurguen Habermas (1993), Michel Onfray (2008), Norbert Elias (1994), Humberto Maturana (2004) entre otros.

Pascuali (2007) entiende a la comunicación intersubjetiva como otra forma de comunicación humana. Su teoría se sustenta en la interacción a través de símbolos y signos, lo que se conoce como interaccionismo simbólico. Este fenómeno acuñado por Herbert 
Blumer (1986) considera que las personas actúan sobre los objetos e interactúan con otras personas a partir de los significados de los objetos, es decir, a partir de símbolos. Aquí los significados son producto de la interacción social, principalmente de la comunicación. Finalmente, las personas seleccionan, organizan, reproducen y transforman los significados en los procesos interpretativos en función de sus expectativas y propósitos.

La teoría de Pascuali (2007) menciona que la intersubjetividad requiere de interacción, intercambio, y en toda situación de interacción o intercambio se produce un contacto intersubjetivo. Es decir, la comunicación como la intersubjetividad son conceptos interdependientes. Para la sociología fenomenológica cualquier forma de interacción social tiene su origen en las construcciones en torno a la comprensión del otro. Por simple que sea, cualquier interacción entre sujetos presupone una serie de construcciones de sentido común; en este caso, se construye la conducta que un sujeto prevé de otro, y viceversa. Los significados sociales, por tanto, no permanecen o se hallan en los objetos físicos, sino en las relaciones de los sujetos entre ellos mismos, y entre ellos y los objetos del mundo de la vida cotidiana (Pech, Rizo, \& Romeu, 2008).

Son estos significados compartidos y transmitidos mediante la interacción de los sujetos, lo que permite un nivel de entendimiento entre éstos y adicionalmente se van construyendo nuevas formas de relacionarse con el otro.

A través de la acción comunicativa propuesta por Habermas (1993), los sujetos pueden desarrollar y mejorar su capacidad de entendimiento y comunicación. Este concepto de comunicación intersubjetiva comparte el objeto empírico de la comunicación interpersonal, pero se diferencia de ella en perspectiva, destacando -aquella- la construcción social inherente al fenómeno. La comunicación intersubjetiva en esta teoría se relaciona con la posibilidad de intercambio de perspectivas apuntando a la construcción social de un mundo compartido: el mundo de la vida.

La comunicación intersubjetiva es la base para la construcción de los significados sociales, orientada al entendimiento y a la compresión e, idealmente, posibilitadora de los consensos necesarios que permitirían, en último término, un tejido social democrático basado en argumentos racionales propios de hombre libres que actúan por el bien colectivo (García, 2013, pp. 115).

La Asociación Mexicana de Investigadores de la Comunicación (AMIC), entre sus miembros, tienen tres fuertes enfoques: el Filosófico, la Sociología y la Psicología Social. Su concepto aún mantiene problemas en la diferencia entre la comunicación interpersonal y la comunicación intersubjetiva en lo que respecta al papel de las emociones.

En la otra orilla y desde una perspectiva comunicacional, encontramos a los comunicadores de la Universidad de Rosario situada en Argentina, esta perspectiva comunicacional que integra a la intersubjetividad como una de sus troncos científicos donde sostiene la teoría de la Comunicación Estratégica propuesta por Sandra Massoni (2003), pero se aparta en dos aspectos de las teorías anteriormente expuestas: en primer lugar presenta una teoría de la intersubjetividad no dualista que entiende a la comunicación como un fenómeno complejo, situacional y fluido (Massoni, 1990 y 2003), desconectándose así de la producción, circulación y consumo de mensajes. Esta teoría busca analizar la multidi- 
mensionalidad del fenómeno comunicacional para operar transformaciones en las configuraciones actuales de las modalidades de vinculación intersubjetiva. Su objetivo es "propiciar el encuentro en la diversidad como un cambio social conversacional que enactúa" (Massoni, 2003). Propone rebasar las investigaciones típicas de la investigación tradicional en ciencias sociales, en tanto son formas representacionalistas, escindidas y dualistas, y por lo mismo vacías de comunicación, se sustenta en la teoría de la comunicación que aborda el encuentro sociocultural (Massoni, 2003) comprensiva y aplicable de modo general.

En segundo lugar, realiza un desplazamiento de la ilusión de “yo" a las Matrices Socioculturales como auto-dispositivos colectivos, se interesa en enfocar el recorte comunicacional, trabajando en las particulares modalidades de vínculo que las Matrices Socioculturales mantiene con diferentes componentes del problema. En esta modalidad se aleja de las investigaciones clásicas en cuanto no interesa mirar las características de los grupo y sectores en términos de hay/no hay; tampoco se afana por caracterizar en ellos los componentes de ausente/presente del sistema, sino más bien en hacer un aporte en el entorno la especificación de las características que asume la relación con el problema y a la vez sus consecuencias en la configuración de las identidades colectivas. Aclara además que:

La idea de un abordaje multiparadigmático responde a la búsqueda de un conocimiento que no se desentienda de los acoplamientos dinámicos y evolutivos de la realidad y los sujetos. Proponemos este enfoque a partir de reconocer en los paradigmas capacidades para abordar diferentes dimensiones de lo real, y a la vez sus aportes específicos en la genealogía de esas mismas realidades, sin dejar de recuperar esta operación de construcción/deconstrucción, para enfatizar la acción transformadora de la ciencia (Massoni, 2001, p. 2).

En este sentido Massoni (2003) instala a la teoría de la intersubjetividad como una subsidiaria de la teoría de la complejidad de Edgar Morín (1999), esta se dirigió a poner de relieve los aspectos dimanantes de la subjetividad humana mediante la obtención de un saber acerca de lo que las personas viven, perciben, sienten, piensan y expresan en diversas situaciones de su vida cotidiana diaria. Busca alcanzar un saber acerca del proceso de constitución de sentidos para la intersubjetividad de esas personas, así como de la interpretación de estos sentidos por ellas, a partir de sus propias acciones y por el significado que éstas les asignan a las mismas (Sotolongo, 2000).

Esta teoría de la intersubjetividad jerarquiza al sujeto por encima del objeto. A diferencia de la epistemológica clásica que mira la relación sujeto-objeto, como un objeto-fenómeno (subjetivado) de la experiencia de la conciencia. Por lo tanto, convierte, de hecho, a la relación sujeto-objeto en una relación entre dos subjetividades - un sujeto y algo subjetivado por éste-. De ahí que, con justicia también, se le considere como subjetivante.

Esta teoría considera que en el espacio de la comunicación no hay sólo producción de un proceso, sino también, y principalmente, producción de una trama ontológica que va generando un tipo de conocimiento. Es sustancial reconocer que el otorgar sentido de la mediación no es algo que se hace de una vez y para siempre, es algo que se hace a cada momento, pero no en cualquier dirección sino en base a y desde una cierta lógica 
de funcionamiento -matriz sociocultural- que se reconoce actuando en una situación de comunicación organizada a partir de una racionalidad dominante (Massoni, 2011).

El deporte es uno de los temas que no obstante la mirada de la mayoría de investigadores científicos, sin embrago, existe una cantidad interesante de estudiosos que abordan el tema desde diferentes perspectivas: El deporte como una forma de desarrollo económico, en este línea encontramos a investigadores como Gómez (2011), Filon (2009), Marca (2009), Rodríguez (2000), Salvador (2003), Castellanos (2001), entre otros; en la perspectiva del deporte como turismo tenemos, entre otros: Nogueras, Medina \& Sánchez (2004, 2005), Lipovetsky \& Charles (2006), Lipovestky (2007), Beck (1998), Bauman, (2000), Merino (1998); dentro de los investigadores que estudian al deporte como ocio encontramos a García (1998), San Salvador (1998) este último autor clasifica las dimensiones del ocio ejemplificando cada una de ellas con su particular práctica deportiva y un tipo específico de turismo. Por ejemplo, a la dimensión festiva, el deporte autóctono y el turismo cultural, a la dimensión solidaria, el voluntariado deportivo y el turismo social, a la dimensión ecológica, las actividades físico deportivas en la naturaleza, a la dimensión terapéutica, el deporte salud y el turismo terapéutico y más; el deporte como competencia y como salud es considerada como una herramienta clave en la promoción de la salud y en la prevención de los efectos negativos asociados a un estilo de vida sedentario.

En la mayoría de sociedades, el deporte amateur, en el ámbito de los clubes, goza de un gran apoyo público y se considera un escenario idóneo para la integración social. El deporte de élite ocupa una posición concreta en el ámbito público, y sirve como entretenimiento, como medio de construcción de identidad y, hasta cierto punto, como ideal moral (Cachorro, 2007).

Tradicionalmente, la competencia en el campo del deporte y la educación física se ha basado en la experiencia y en los modelos de buenas prácticas. No obstante, desde la década de los 60, el aumento de la concienciación política, por lo que respecta a los beneficios del deporte y la educación física de carácter sociocultural y para la salud, ha llevado al aumento de la investigación y la práctica basada en las evidencias (Renson, 1989). El estudio del juego, el deporte para todos, la educación física, el deporte de élite, el entrenamiento y el ejercicio, la danza e incluso la educación al aire libre se han convertido en un campo académico profesional conocido, en términos generales, como ciencia del deporte; otra especificidad de abordaje es el deporte como alternativa en los procesos cognitivos, es aquí donde nos detendremos para realizar una exploración más exhaustiva.

El deporte en proceso cognitivos ha tenido deferentes perspectivas de abordaje; como procesos de subjetivación (Cachorro, 2010; Scarnatto, 2010; Gumbrecht, 2005; Díaz, 2009), estas investigaciones realizan el seguimiento de estos procesos como instancia de incorporación de reglas, principios y comportamientos deportivos en el mundo interno del sujeto, considerando que es dinámico, relacional y subjetivo, deriva en surcos biográficos diferentes; como prácticas corporales y subjetividad, su finalidad es el estudio del cuerpo como figura social como un tejido de personas interdependientes, ligadas entre sí en varios niveles y de varias maneras (Elías y Dunning, 1996; Chaves, 2004; Ruiz, 1995; Gumbrecht, 2005, Ulrich, 2005; Parlebas, 2002; entre otros); otro tipo de abordaje es familia y participación en actividades deportivas durante la adolescencia (Ramos, Rivera, Moreno y Jiménez-Iglesias, 2011; Strong et al., 2005), ya que la incorporación de la actividad física 
y deportiva regular en la vida del adolescente se asocia no sólo con estilos de vida más saludables durante esta etapa sino también a la construccion de subjetividades (Castillo, Balaguer y García-Merita, 2007; Ianotti, Kogan, Janssen y Boyce, 2009).

Las investigaciones sobre construccion de identidad en espacios deportivos/comunitarios es otra importante perspectiva (Fernández, 1994; Villamayor, 2005, entre otros) en estos espacios los ciudadanos realizan prácticas de comunicación para la producción de sentido y la conformación de subjetividades, estos estudios analizan las practicas que posibilitaran interpretar el escenario que describe como sujetos colectivos.

Otro aspecto es la motivación que receptan los ciudadanos en la realización de actividad física. En este tipo de estudio se ha encontrado que género y edad son dos factores relevantes en la frecuencia de realización de actividad física, al tiempo que se ha observado que la autoeficacia y la competencia percibida, así como el disfrute asociado a la práctica deportiva son factores psicológicos de gran relevancia (Biddle, Whitehead y Nevill, 2005). En este sentido, parece observarse que el modelado de otros significativos, entre los que han sido destacados la familia y el grupo de iguales, ejerce también una influencia fundamental (Escartí y García, 1994; Gil de Montes et al., 2007; Wold y Andersen, 1992), aunque estos estudios no están exentos de contradicciones. Sallis, Prochaska y Taylor (2000), en una revisión sistemática de los estudios publicados sobre variables influyentes en la actividad física de los adolescentes, no encontraron relaciones consistentes entre las variables sociales y la realización de actividad física. Igualmente, sus hallazgos fueron contradictorios con algunos de los alcanzados en trabajos anteriores en lo referente a la influencia de las variables psicológicas y el nivel socioeconómico.

$\mathrm{Al}$ abordar la intersubjetividad y deporte es un destino que pasa por la genealogía del cuerpo y es inevitable no mencionar a Jesús Galindo Cáceres $(2005,2006,2009)$. El autor explora al cuerpo como un código que puede conocerse, escribirse y ser reescrito. La subjetividad social general -moral, política, religiosa- se fragmenta, los individuos pueden decidir sobre su cuerpo incluso a nivel genético.

Galindo (2009) establece que la historia social del cuerpo pasa por la mediación de diversas instituciones e ideologías como la cárcel, el hospital, el manicomio, la escuela, la fábrica, y las mismas formas de la ciudad, de las casas, de los dormitorios. Considera que el territorio físico de lo social corresponde palmo a palmo con el territorio físico de las formas de intervención sobre el cuerpo. En su análisis menciona a la educación como uno de los principales dispositivos de control y divide su interpretación entre la educación física y su contraste entre la educación escolar no física. La educación escolar física trata de poner en forma a la parte móvil del cuerpo y por otra el cuerpo se inmoviliza en la educación escolar intelectual. Las dos figuras son convergentes, partes de una misma visión, de una misma construcción subjetiva social del cuerpo.

El movimiento supone conceptos de educación integral, de educación en sociedad, en forma comunitaria, de educación como forma de comunicación, de educación como ciudadanización.

La educación física ya no desea ser motivo de exclusión social sino de todo lo contrario, se vuelve científica, pero no sólo en un sentido médico, sino en un sentido sociológico, psicológico, comunicológico. Se construye desde una epistemología de punta, la Epistemología Genética. En el tema comunicacional, en un principio se perciben a los cuerpos como 
portadores de los Sistemas de Información son agentes de la comunicación humana. El cuerpo es el primer lugar de los sistemas de información en un sentido fenomenológico y el agente visible de los Sistemas de Comunicación. Desde el cuerpo observamos lo social, son cuerpos en movimiento, cuerpos en relación, cuerpos en lucha, cuerpos en encuentro, cuerpos en búsqueda.

El programa de investigación se plantea sobre Educación Física, donde el cuerpo se asociados a otros cuerpos como sistemas de información que forman sistemas de comunicación, a través de las redes sociales, las formas interpersonales de la vida social, la figura de los cuerpos en relación como figuras constructivas de la comunicación, como figuras construidas por la comunicación (Galindo; 2005, 2006 y 2009).

El presente recorrido sobre la cuestión sobre los procesos intersubjetivos utilizados por organizaciones sociales con intenciones de participación en espacios deportivos habilita concluir que las investigaciones realizadas hasta hoy, no han considerado estas articulaciones. Es por eso, que este proyecto avizora que los procesos intersubjetivos utilizados como estrategia de comunicación para la convocatoria en actividades deportivas es una forma estratégica y novedosa de propiciar la participación en eventos deportivos con vistas de transformación social, en el campo de la salud, que no ha sido analizada por la comunicación.

\section{Matriz de datos de la comunicación enactiva para la inteligibilidad de procesos intersubjetivos en espacios deportivos}

La Matriz Sociocultural con la que trabajaremos en este proyecto es el esquema básico que describe los rasgos principales de la lógica de funcionamiento de un grupo social. Una matriz sociocultural programa en cada grupo su sistema de percepción-acción. Incluye no sólo las condiciones, sino la percepción que cada grupo tiene de ellas -lo simbólico y lo material imbricados-. Este concepto nos permite introducir la problemática del placer en el marco de las determinaciones socioculturales: si alguien hace algo es porque hay una ganancia de algún tipo. La pulsión y la tensión entran en juego.

Con el concepto Matrices Socioculturales encontramos una genealogía en la historicidad de los vínculos. Es decir, una manera de trabajar las relaciones que conservan cierta estabilidad en los sectores o grupos socioculturales como formas que dan cuenta de una trayectoria -más allá de tamaño, cantidad, etc.- Esta es la principal diferencia con los enfoques tradicionales de los estudios socioeconómicos y de marketing, que generalmente trabajan a partir de una descripción previa a la situación que se aborda, estática y prescriptiva de la comunicación. Trabajar con matrices socioculturales como dispositivo de mediaciones supone abordar la comunicación desde lo contingente y lo situacional.

Los actores no son previos al análisis, sino que emergen del recorte operado en función del objetivo de la investigación. Las diferencias no existen en sí mismas, sino que se especifican. Así, el plus de la idea de mediación como categoría llave de la dimensión comunicativa tiene que ver con permitirnos trabajar la transformación desde las relaciones. Es en las relaciones donde sujetos, prácticas e imaginarios se reinventan; y es a partir de las relaciones que se fijan posiciones de diferencia, siempre inestables, pero no aleatorias, entre 
actores sociales. La mediación es un espacio de articulación, no un espacio mediador. No hay extremos entre los cuales mediar; hay relaciones entre matrices que configuran posiciones de sujetos. Descubrir estos espacios nos permite trabajar para la transformación en el sitio privilegiado de su constitución.

La propuesta de la matriz de datos de Investigación Enactiva en Comunicación (IEC) para la lectura de los procesos intersubjetivos en eventos deportivos como un dispositivo intersubjetivo estratégico es habilitada por la implementación del proyecto típico de IEC: Versión Técnica Comunicacional (VTC) con sus componentes, niveles, subniveles y aspectos; sumado a las técnicas Marcas de Racionalidad Comunicacional (MRC); mapeo comunicacional de actores, reconocimiento, jerarquización y caracterización de las Matrices Socioculturales (MS); el Árbol de Soluciones (AS) e investigación de campo y plan operativo de la estrategia de comunicación. Para necesidades de esta investigación y por cuestiones de espacio, trabajaremos únicamente con la implementación del dispositivo Matrices Socioculturales, únicamente como objetivo de análisis, considerando que el proyecto de investigación enactiva es interconectada, es decir, enactuda. Una vez determinado los metodologías, métodos y herramientas procedemos a realizar el recorte comunicación en el objeto de estudio.

El cantón Sígsig se encuentra ubicada en las sagradas colinas de Los Andes ecuatorianos, pertenece a la provincia del Azuay en la republica del Ecuador. Tiene una población que se acerca a los 30000 habitantes, sus actividades económicas son la agricultura y las artesanías. La artesanía más famosa es el sombrero de paja toquilla, actividad que ostente el título de Patrimonio Cultural de la Humanidad. En el tema turístico su punto más importante es el Complejo Arqueológico de Chopshi, lugar que alberga ruinas de comunidades ancestrales con más de 7000 años de antigüedad, es el territorio del Cacique Duma.

En el tema social, el cantón Sígsig cuenta con las Jornadas Deportivas Vacacionales (JDV) considerado como evento deportivo más constante y antigua del Ecuador con una tradición que se acerca a conmemorar los 50 años de actividad deportiva y social. Las Jornadas Deportivas Vacaciones de Sígsig nace el año 1972 con la participación 8 equipos integrados por sigseños y un total de 300 deportistas. En la actualidad las JDV de Sígsig está integrada por 11 clubs y 1500 deportistas, entre niños, niñas, adolescentes, senior y post 40 años.

Las Jornadas Deportivas Vacacionales de Sígsig son organizadas año tras año y se desarrolla entre la última semana de agosto y la primera semana de septiembre. La organización es desarrollada por un club, por año y el turno se designa en base a un sorteo. La máxima autoridad de las Jornadas Deportivas Vacacionales de Sígsig es el Comité Rector, integrado por un miembro de cada club y el presidente de Liga Deportiva Cantonal de Sígsig. Este comité es el encargado de sancionar a los clubs que rompan las reglas establecidas desde hace más de 40 años y resuelven temas en conflicto.

Las JDV de Sígsig es un evento deportivo que reúne a más de 10.000 personas que llegan a residir en el cantón durante la semana determinada para el evento deportivos y alrededor de 5000 visitantes que acuden: A la inauguración, miércoles de peña artística y finales de básquet, futbol y vóley. En las dos últimas versiones se registra la participación de 1500 deportistas en representación de 11 clubes -Dangers, Estudiantes SRC, Liga Parroquial de Cutchil, Liga Parroquial de Gûel, Llactazhungu, Profesionales, San Sebastián, Sauces, Siete 
Estrellas, Sigsales y SigsigSporting-, en las disciplinas deportivas de futbol, básquetbol, indor, ecuavolley, atletismo, ciclismo, tenis de mesa, ajedrez, entre otros. Estas disciplinas se disputan en categorías de menores de 12 años, 15 años, 19 años, sénior y post 40 años, es decir, toda una fiesta familiar. La promoción del evento, si bien se promociona en los medios de comunicación de cobertura regional, estas son meramente invitación para los turistas, mientras que los deportistas, barras de equipos y dirigentes trabajan todo el año para que todo sea perfecto. En el tema de los premios para los ganadores de cada competencia, no existe dinero de por medio, al club ganador le entregan únicamente un trofeo que no supera los 300 dólares, sin embargo, los gastos que incurren cada disciplina sobrepasan los 1500 dólares. Para solventar el tema económico que requieren los clubs para cada categoría, sus miembros se dedican a realizar sorteos y rifas durante todo el año, en definitiva, es un evento en donde solo reina el deporte.

Un paso previo a la aplicación de la estrategia de comunicación como dispositivo doble, es establecer las técnicas y herramientas a implementar para cumplir con los objetivos propuestos en esta investigación. A continuación colocamos la matriz de datos de la IEC, con la cual podremos visualizar el recorte comunicacional que implementamos en nuestra estrategia comunicacional como dispositivo de inteligibilidad y de interpelación.

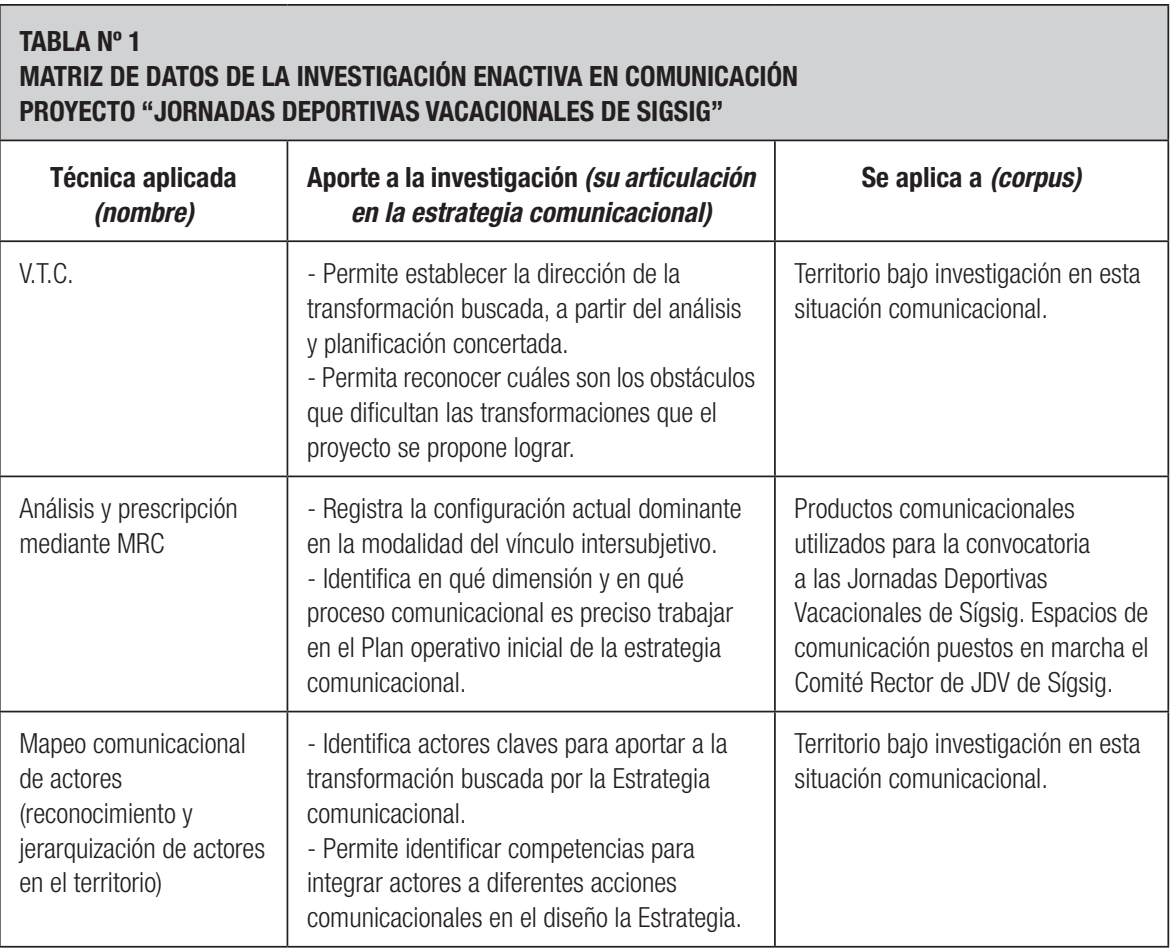




\begin{tabular}{|c|c|c|}
\hline Caracterización de MS & $\begin{array}{l}\text { - Describe los rasgos principales de la lógica } \\
\text { de funcionamiento de un grupo o sector } \\
\text { social, su linaje de transformaciones. }\end{array}$ & $\begin{array}{l}\text { Territorio bajo investigación en esta } \\
\text { situación comunicacional. }\end{array}$ \\
\hline $\begin{array}{l}\text { Análisis de fuentes } \\
\text { documentales }\end{array}$ & $\begin{array}{l}\text { - Permite entender, describir y representar } \\
\text { los documentos de forma unificada } \\
\text { sistemática para facilitar su recuperación. } \\
\text { Entendiéndola como un soporte material de } \\
\text { hechos, fenómenos y manifestaciones de la } \\
\text { realidad social. }\end{array}$ & Tesis, libros, revistas científicas. \\
\hline $\begin{array}{l}\text { Análisis de fuentes } \\
\text { estadísticos }\end{array}$ & $\begin{array}{l}\text { - Permitirá mirar la evolución de la } \\
\text { participación de deportistas, turistas y } \\
\text { espectadores en las Jornadas Deportivas } \\
\text { Vacacionales de Sígsig durante los periodos } \\
\text { en cuestión. }\end{array}$ & Tesis, libros, revistas científicas. \\
\hline Entrevista Etnográfica & - Permite la obtención de testimonios & Deportistas, Turistas y espectadores \\
\hline $\begin{array}{l}\text { Test de Trasposición } \\
\text { temporal }\end{array}$ & $\begin{array}{l}\text { - Permite ver las conceptualizaciones del } \\
\text { problema en distintos periodos relacionados. }\end{array}$ & $\begin{array}{l}\text { Deportistas, Turistas y } \\
\text { espectadores. }\end{array}$ \\
\hline $\begin{array}{l}\text { Test de modos } \\
\text { y espacios de } \\
\text { comunicación }\end{array}$ & $\begin{array}{l}\text { - Permite mirar las formas de conocer, } \\
\text { innovaciones en torno a la problemática y las } \\
\text { formas habituales de esos contactos }\end{array}$ & $\begin{array}{l}\text { Deportistas, Turistas y } \\
\text { espectadores. }\end{array}$ \\
\hline $\begin{array}{l}\text { Test de jerarquización de } \\
\text { actores y competencias }\end{array}$ & $\begin{array}{l}\text { - Identifica actores claves para aportar a la } \\
\text { transformación buscada por la Estrategia } \\
\text { comunicacional. } \\
\text { - Permite identificar competencias para } \\
\text { integrar actores a diferentes acciones } \\
\text { comunicacionales en el diseño la Estrategia. }\end{array}$ & $\begin{array}{l}\text { Territorio bajo investigación en esta } \\
\text { situación comunicación, Comité } \\
\text { Rector, club, turistas y espectadores } \\
\text { que trabajan con el tema deportivo }\end{array}$ \\
\hline $\begin{array}{l}\text { Test de articulaciones y } \\
\text { modalidad de vínculo }\end{array}$ & $\begin{array}{l}\text { - Registra áreas de la cadena de valor } \\
\text { de mayor impacto para la articulación de } \\
\text { políticas sectoriales. }\end{array}$ & Comité Rector de JDV de Sígsig. \\
\hline
\end{tabular}

Fuente: Elaboración de los autores en base a Massoni (2013).

De lo anotado en la parte superior, iniciamos con la descripción de la aplicación del dispositivo Matrices Socioculturales.

La caracterización de Matrices Socioculturales (MS) es una técnica del Proyecto de Investigación Enactiva en Comunicación que especifica la operación cognitiva de la estrategia comunicacional: el Reconocimiento de los auto dispositivos colectivos (Massoni, 2003). Utiliza un conjunto de procedimientos estandarizados de investigación mediante los cuales registra y considera una serie de datos de interés comunicacional en el marco de la ciencia de la articulación: establece el reconocimiento de modalidades diferenciales y flui- 
das de vinculación intersubjetiva micro-macrosocial en torno al problema comunicacional en el territorio de la investigación y en torno al tema que se investiga.

En esta ocasión no se desarrolló un gabinete por el tema de la pandemia, sin embargo, se desarrolló una sesión de trabajo, en algún lugar digital entre Sígsig y Cuenca, Ecuador y Toronto, Canadá, con la presencia del presidente del Comité Rector de JDV de Sígsig el señor René Astudillo, con Lorena y Klever en calidad de comunicadores/investigadores, caber recalcar que uno de los autores Klever fue organizador de un evento de JDV de Sígsig en el año 2013.

La conformación de las muestras para caracterizar a cada una de las Matrices Socioculturales definidas en la investigación en comunicación estratégica, se determinó siguiendo el criterio de máxima heterogeneidad entre los tipos de entrevistados de cada grupo identificados como protagónicos en la Versión Técnica Comunicacional.

La muestra elegida fue muestreo por cuotas para seleccionar las Matrices Socioculturales porque permite analizar los tipos de grupos o sectores y comparar ciertas secciones de la población que exhiben determinadas características.

\begin{tabular}{|l|l|}
\hline $\begin{array}{l}\text { TABLA No } 2 \\
\text { Reconocimiento de Matrices Socioculturales del proyecto Jornadas } \\
\text { Deportivas Vacacionales de Sígsig. }\end{array}$ & $\begin{array}{l}\text { HERRAMIENTA No } 2 \\
\text { Agosto/2020 }\end{array}$ \\
\hline Los que organizan: Miembros del Comité Rector & \\
\hline Los que asisten: Ciudadanos del cantón Sígsig. & \\
\hline Los que participan: Deportistas de los 11 clubs (locales y extranjeros) \\
\hline Los que colaboran: Cuerpo de Bomberos, Hospital de Sígsig, Cruz Roja, Policía Nacional en el cantón. \\
\hline $\begin{array}{l}\text { Los que coordinan: GAD de Sígsig, Prefectura del Azuay, Federación Deportiva del Azuay, Liga Deportiva Cantonal } \\
\text { de Sígsig y Las Ligas Parroquiales correspondientes. }\end{array}$ \\
\hline Los que auspician: Municipio de Sígsig, Banco del Pichincha, Cooperativa JEP, Cooperativa Jardín Azuayo. \\
\hline Los que visitan: Turistas que residen en casas del centro urbano, durante la semana de JDV. \\
\hline
\end{tabular}

Fuente: Elaboración de los autores en base a Massoni (2013).

Luego de identificar las Matrices Socioculturales (MS) se procedió a recuperar saberes, intereses, necesidades y expectativa de los grupos involucrados para organizar en la estrategia comunicacional acciones capaces de asumir las lógicas en juego (Massoni, 2007). Las Matrices Socioculturales (MS) no se elaboran, solo se reconocen como un linaje de acciones compartidas por un grupo o sector social que implica una particular modalidad de vínculo con la problemática. En esta perspectiva, el abordaje situacional permitió reconocer a lo material y lo simbólico imbricados, no escindidos. 


\begin{tabular}{|c|c|c|c|}
\hline \multicolumn{3}{|c|}{$\begin{array}{l}\text { TABLA N }{ }^{\circ} 3 \\
\text { Resultado de la Matriz de datos de la Investigación Enactiva en } \\
\text { Comunicación proyecto Jornadas Deportivas Vacacionales de Sígsig. }\end{array}$} & $\begin{array}{l}\text { HERRAMIENTA No3 } \\
\text { Agosto/2020 }\end{array}$ \\
\hline \multicolumn{4}{|c|}{ Componente del problema: Todos } \\
\hline Actores/Matrices & $\begin{array}{l}\text { Intereses/ } \\
\text { Necesidades }\end{array}$ & $\begin{array}{l}\text { Producto, } \\
\text { espacio, proceso } \\
\text { comunicacional }\end{array}$ & Resultado \\
\hline $\begin{array}{l}\text { Miembros del Comité } \\
\text { Rector / Los que } \\
\text { organizan }\end{array}$ & $\begin{array}{l}\text { Obtener lo } \\
\text { reconocimiento de sus } \\
\text { gestiones/ Obtener } \\
\text { resultados visibles y } \\
\text { reconocimientos sociales } \\
\text { por los ciudadanos } \\
\text { (hacer bien las cosas). }\end{array}$ & $\begin{array}{l}\text { Comunicación personal. } \\
\text { Medios digitales: } \\
\text { Dimensión del dato. }\end{array}$ & $\begin{array}{l}\text { Los espacios, medios y } \\
\text { procesos no muestran } \\
\text { una innovación en la } \\
\text { comunicación. }\end{array}$ \\
\hline $\begin{array}{l}\text { Ciudadanos del cantón } \\
\text { Sígsig/ Los que asisten. }\end{array}$ & $\begin{array}{l}\text { Visibilizaron ante la } \\
\text { sociedad, diversión y } \\
\text { acuden a un encuentro } \\
\text { con la identidad local. }\end{array}$ & $\begin{array}{l}\text { Acuden al encuentro con } \\
\text { sus amigos y conocidos } \\
\text { (los que se fueron a } \\
\text { residir en otros lugares } \\
\text { por diferentes motivos): } \\
\text { Dimensión del Encuentro } \\
\text { Sociocultural. }\end{array}$ & $\begin{array}{l}\text { Los asistentes sigseños } \\
\text { acuden a un encuentro } \\
\text { que es parte de } \\
\text { su historia, donde } \\
\text { encuentra a amigos de } \\
\text { la infancia. }\end{array}$ \\
\hline $\begin{array}{l}\text { Deportistas de los } \\
11 \text { clubs (locales y } \\
\text { extranjeros): Los que } \\
\text { participan. }\end{array}$ & $\begin{array}{l}\text { Locales: Visibilidad y } \\
\text { reconocimiento Social/ } \\
\text { mejorar su salud y su } \\
\text { aspecto físico, encuentro } \\
\text { con sus raíces. } \\
\text { Extranjeros: viven al } \\
100 \% \text { sus partidos, } \\
\text { se sienten parte } \\
\text { de los locales y los } \\
\text { movimientos en la } \\
\text { cancha son exaltados } \\
\text { por los asistentes y } \\
\text { aprendido por sus } \\
\text { compañeros y rivales, se } \\
\text { sientes importantes por } \\
\text { sus conocimientos. }\end{array}$ & $\begin{array}{l}\text { Acuden al encuentro } \\
\text { con las personas que } \\
\text { viven y se apasionan por } \\
\text { el deporte: Encuentro } \\
\text { Sociocultural. }\end{array}$ & $\begin{array}{l}\text { Los extranjeros en un } \\
\text { primer momento acuden } \\
\text { al encuentro por dinero, } \\
\text { pero al siguiente año } \\
\text { quieren ir porque se } \\
\text { sienten bien y no tienen } \\
\text { presión por la obtención } \\
\text { de los resultados, lo } \\
\text { hacen por que les nace, } \\
\text { porque quieren ser parte } \\
\text { del encuentro como uno } \\
\text { más de los locales. }\end{array}$ \\
\hline $\begin{array}{l}\text { Cuerpo de Bomberos, } \\
\text { Hospital de Sígsig, Cruz } \\
\text { Roja, Policía Nacional } \\
\text { en el cantón: Los que } \\
\text { colaboran. }\end{array}$ & $\begin{array}{l}\text { Asisten al encuentro } \\
\text { como un espectador } \\
\text { más, un ciudadano más, } \\
\text { pero con la mirada en } \\
\text { sus responsabilidades } \\
\text { y listos para atender las } \\
\text { emergencias. }\end{array}$ & $\begin{array}{l}\text { Acuden al encuentro } \\
\text { con las personas que } \\
\text { viven y se apasionan por } \\
\text { el deporte: Encuentro } \\
\text { Sociocultural. }\end{array}$ & $\begin{array}{l}\text { Utilizan al encuentro } \\
\text { de la misma forma que } \\
\text { los espectadores, pero } \\
\text { con la responsabilidad } \\
\text { atientas. }\end{array}$ \\
\hline
\end{tabular}




\begin{tabular}{|c|c|c|c|}
\hline $\begin{array}{l}\text { GAD de Sígsig, } \\
\text { Prefectura del Azuay, } \\
\text { Federación Deportiva del } \\
\text { Azuay, Liga Deportiva } \\
\text { Cantonal de Sígsig y } \\
\text { Las Ligas Parroquiales } \\
\text { correspondientes: Los } \\
\text { que coordinan. }\end{array}$ & $\begin{array}{l}\text { Obtener lo } \\
\text { reconocimiento de sus } \\
\text { gestiones/ Obtener } \\
\text { resultados visibles y } \\
\text { reconocimientos sociales } \\
\text { por los ciudadanos } \\
\text { (hacer bien las cosas). }\end{array}$ & $\begin{array}{l}\text { Comunicación personal. } \\
\text { Medios digitales: } \\
\text { Dimensión del dato. }\end{array}$ & $\begin{array}{l}\text { Los espacios, medios y } \\
\text { procesos no muestran } \\
\text { una innovación en la } \\
\text { comunicación. }\end{array}$ \\
\hline $\begin{array}{l}\text { Municipio de Sígsig, } \\
\text { Banco del Pichincha, } \\
\text { Cooperativa JEP, } \\
\text { Cooperativa Jardín } \\
\text { Azuayo: Los que } \\
\text { auspician. }\end{array}$ & $\begin{array}{l}\text { Obtener lo } \\
\text { reconocimiento de } \\
\text { sus clientes/ obtener } \\
\text { visibilidad ante sus } \\
\text { clientes y potenciales } \\
\text { clientes y lo consideran } \\
\text { como un aporte a la } \\
\text { responsabilidad social. }\end{array}$ & $\begin{array}{l}\text { Comunicación personal. } \\
\text { Medios digitales: } \\
\text { Dimensión del dato. }\end{array}$ & $\begin{array}{l}\text { Los espacios, medios y } \\
\text { procesos no muestran } \\
\text { una innovación en la } \\
\text { comunicación. }\end{array}$ \\
\hline $\begin{array}{l}\text { Turistas que residen en } \\
\text { casas del centro urbano, } \\
\text { durante la semana de } \\
\text { JDV: Los que visitan. }\end{array}$ & $\begin{array}{l}\text { Espectar el más técnico, } \\
\text { apasionado y vívido } \\
\text { evento deportivo del } \\
\text { país. Observar como la } \\
\text { familia sigseña se reúne } \\
\text { a través del deporte } \\
\text { (descansar y conocer a } \\
\text { gente interesante). }\end{array}$ & $\begin{array}{l}\text { Acuden al encuentro } \\
\text { con las personas que } \\
\text { viven y se apasionan por } \\
\text { el deporte: Encuentro } \\
\text { Sociocultural. }\end{array}$ & $\begin{array}{l}\text { Acuden al encuentro } \\
\text { sociocultural por su } \\
\text { pasión por el deporte } \\
\text { que tiene identidad } \\
\text { propia. }\end{array}$ \\
\hline $\begin{array}{l}\text { Análisis de fuentes } \\
\text { documentales }\end{array}$ & & $\begin{array}{l}\text { En la revisión de tesis, } \\
\text { libros, reglamentos y } \\
\text { revistas científicas. }\end{array}$ & $\begin{array}{l}\text { Las tesis, libro, y } \\
\text { revistas que tienen } \\
\text { relación a las JDV de } \\
\text { Sígsig cumplen una } \\
\text { función de información } \\
\text { y se mantienen en el } \\
\text { dimensión del Dato, sin } \\
\text { embargo, en la sección } \\
\text { V, artículo } 15 \text { numeral } \\
15 \text { del Reglamento } \\
\text { de JDV de Sígsig } \\
\text { que dice lo siguiente: } \\
\text { podrán inscribirse para } \\
\text { participar en las JDV de } \\
\text { Sígsig quienes cuenten } \\
\text { con los siguientes } \\
\text { requisitos: Ser nacido } \\
\text { (a) en el cantón Sígsig. } \\
\text { Ser hijo (a), nieto (a) o } \\
\text { bisnieto (a) de padre o } \\
\text { madre sigseños. }\end{array}$ \\
\hline
\end{tabular}




\begin{tabular}{|c|c|c|}
\hline & & $\begin{array}{l}\text { Cónyuge de un sigseño } \\
\text { (a) de nacimiento. Ser } \\
\text { cónyuge de hijo (a), nieto } \\
\text { (a) o bisnieto (a) de padre } \\
\text { o madre sigseños. Quien } \\
\text { este domiciliado (a) en } \\
\text { el cantón por más de } 2 \\
\text { años (con informe de } \\
\text { situación sumaria). Este } \\
\text { artículo funciona como } \\
\text { vínculo intersubjetivo } \\
\text { de donde se propicia el } \\
\text { encuentro sociocultural } \\
\text { conversacional que } \\
\text { enactua. }\end{array}$ \\
\hline $\begin{array}{l}\text { Análisis de fuentes } \\
\text { estadísticos }\end{array}$ & $\begin{array}{l}\text { Análisis de estadísticas } \\
\text { archivadas en el Comité } \\
\text { Rector de Jornadas } \\
\text { Deportivas Vacacionales } \\
\text { de Sígsig }\end{array}$ & $\begin{array}{l}\text { En la primera jornada } \\
\text { deportiva vacacional de } \\
1972 \text { se inició con } 7 \\
\text { club y } 200 \text { deportistas } \\
\text { en la última edición } \\
\text { realizada en } 2019 \\
\text { se registraron } 1500 \\
\text { deportistas en sus } 11 \\
\text { club asistentes. }\end{array}$ \\
\hline Etnográfica digital. & $\begin{array}{l}\text { Deportistas, Turistas y } \\
\text { espectadores. }\end{array}$ & $\begin{array}{l}\text { Hacen hincapié en lo } \\
\text { más importante que les } \\
\text { convoca al encuentro es } \\
\text { que es una competencia } \\
\text { entre familiares y } \\
\text { conocidos donde el } \\
\text { linaje de sangre es lo } \\
\text { más importante para la } \\
\text { unidad de los sigseños. }\end{array}$ \\
\hline $\begin{array}{l}\text { Test de Trasposición } \\
\text { temporal }\end{array}$ & $\begin{array}{l}\text { Deportistas, Turistas y } \\
\text { espectadores. }\end{array}$ & $\begin{array}{l}\text { Miran a las JDV de Sígsig } \\
\text { de } 10 \text { años atrás como } \\
\text { un evento deportivo en } \\
\text { una curva ascendente. } \\
\text { En la actualidad } \\
\text { como un evento en } \\
\text { estancamiento por el } \\
\text { tema del COVID-19 y en } \\
10 \text { años después como } \\
\text { un evento grande con } \\
\text { mayor fortaleza puesto } \\
\text { que los deportistas y } \\
\text { espectadores esperan } \\
\text { con ansias la nueva } \\
\text { edición. }\end{array}$ \\
\hline
\end{tabular}




\begin{tabular}{|l|l|l|l|}
\hline $\begin{array}{l}\text { Test de modos } \\
\text { y espacios de } \\
\text { comunicación }\end{array}$ & $\begin{array}{l}\text { Deportistas, Turistas y } \\
\text { espectadores. }\end{array}$ & $\begin{array}{l}\text { Las formas de } \\
\text { comunicación son } \\
\text { tradicionales y en } \\
\text { medios tradicionales y } \\
\text { apoyados por las nuevas } \\
\text { tecnologías. }\end{array}$ \\
\hline $\begin{array}{l}\text { Test de articulaciones y } \\
\text { modalidad de vínculo }\end{array}$ & & $\begin{array}{l}\text { Deportistas, Turistas y } \\
\text { espectadores. }\end{array}$ & $\begin{array}{l}\text { El vínculo intersubjetivo } \\
\text { está en la sección V, } \\
\text { artículo 15 numeral 15 } \\
\text { del Reglamento de JDV } \\
\text { de Sígsig. }\end{array}$ \\
\hline
\end{tabular}

Fuente: Elaboración de los autores en base a Massoni (2013).

Como se pude visualizar dentro del cuadro superior todos los resultados tienen un vínculo intersubjetivo en la sección V, artículo 15, numeral 15 del Reglamento de JDV de Sígsig, donde se establece que podrán inscribirse quienes cuenten con los siguientes requisitos: Ser nacido (a) en el cantón Sígsig. Ser hijo (a), nieto (a) o bisnieto (a) de padre o madre sigseños. Cónyuge de un sigseño (a) de nacimiento. Ser cónyuge de hijo (a), nieto (a) o bisnieto (a) de padre o madre sigseños. Quien este domiciliado (a) en el cantón por más de 2 años (con informe de situación sumaria). Este artículo funciona como vínculo intersubjetivo de donde se propicia el encuentro sociocultural conversacional que enactua.

\begin{tabular}{|c|c|c|}
\hline \multicolumn{2}{|c|}{$\begin{array}{l}\text { TABLA N }{ }^{\circ} 4 \\
\text { ANALISIS DEL PROCESO INTERSUBJETIVO EN LAS MATRICES } \\
\text { SOCIOCULTURALES. } \\
\text { PROYECTO JORNADAS DEPORTIVAS VACACIONALES DE SIGSIG } \\
\text { PIEZA/ESPACIO COMUNICACIONAL: Proceso intersubjetivo de Linaje }\end{array}$} & $\begin{array}{l}\text { HERRAMIENTA No } 1 \\
\text { Octubre/2017 }\end{array}$ \\
\hline MRC reconocidas & \multicolumn{2}{|c|}{ Recursos comunicacionales que la hacen emerger } \\
\hline $\begin{array}{l}\text { Heterogeneidad y multiplicidad - } \\
\text { Dimensión sociocultural -. }\end{array}$ & \multicolumn{2}{|c|}{$\begin{array}{l}\text { Forma estratégica de convocatoria y participación deportiva -El proceso } \\
\text { intersubjetivo (la comunicación) actúa como articulador en la diversidad } \\
\text { sociocultural-. }\end{array}$} \\
\hline
\end{tabular}

Fuente: Elaboración de los autores en base a Massoni (2013). 


\section{Conclusiones}

Iniciamos el apartado resaltando la importancia de la implementación de la perspectiva científica de investigación estratégica comunicacional en fenómenos sociales donde la dimensión comunicacional es predominante.

Estudiar a los procesos intersubjetivos desde la perspectiva de la Comunicación Enactiva entendiéndola como estrategia de comunicación, implica el examen de un tipo de convocatoria y participación que privilegia la acción simbólica de la comunicación y una estrategia, la cual tiene como propósito conseguir cambios para la optimización del tramado social (Massoni-Pérez, 2009).

La comunicación ya no hace foco en la eficacia del discurso sino en los sentidos enactuados. Como comunicadores estratégicos tenemos la tarea de diseñar estrategias de comunicación como dispositivos del encuentro que permita ponernos cerca de aquello que designamos colectivamente como lo real. La potencia de las estrategias de comunicación radica justamente en la posibilidad de tomar decisiones respecto de cómo aportamos específicamente como comunicadores, a propiciar ese cambio social conversacional, así como lo hace El Comité Rector de JDV de Sígsig.

Entender que un proceso intersubjetivo actuó como un dispositivo estratégico en los espacios públicos del ámbito deportivo y cultural, es mirarla como una estrategia de comunicación para "dirigir el tránsito espacio/temporal desde una situación dada a otra deseada" (Massoni-Pérez, 2009, p. 266).

Este proceso intersubjetivo gestionado por el Comité Rector de JDV no fue efectuado por la transmisión unidireccional de mensajes o prácticas, más bien, se produjo por un proceso dinámico en el que intervinieron y tomaron parte una diversidad de actores implicados. Como podemos observar el proceso intersubjetivo presente en las JDV de Sígsig logró funcionar como estrategia de comunicación porque sus "dimensiones hasta entonces desconectadas, se unieron, trastocaron e hibridaron para generar un algo que no existía previamente, un algo que enactúa porque hizo y hace emerger nuevas realidades" (Massoni-Pérez, 2009, p. 313).

Con esta acción se logra determinar que el proceso intersubjetivo operó y opera como estrategia de comunicación para la convocatoria y participación en espacios públicos del ámbito deportivo y cultural.

Con el concepto Matrices Socioculturales encontramos una genealogía en la historicidad de los vínculos. Es decir, una manera de trabajar las relaciones que conservan cierta estabilidad en los sectores o grupos socioculturales como formas que dan cuenta de una trayectoria (más allá de tamaño, cantidad, etc.), estamos hablando de un proceso intersubjetivo comunicacional basado en el linaje y no en mensajes transmitidos.

En conclusión, el proceso intersubjetivo, de linaje, en este caso, no solo ha actuado y actúa como una forma estratégica de convocatoria y participación en espacios deportivos, sino también: Como espacio de construcción y transmisión de conocimiento de saberes deportivos; como espacio para la re-significación la identidad colectiva; como resignificación del ser sigseño como poseedor de saberes y habilidades deportivos que son reconocidas en las provincias cercanas; como espacio para constituir al territorio como elemento identitario; como un espacio de construcción social donde se produce el trabajo en equipo; un 
elemento para mirar la emergencia de una conversación entre los primeros organizadores y participantes y la actualidad; un espacio para la conformación de organizaciones o asociaciones, es decir, clubs; un lugar para el desarrollo social y cultural, en definitiva, el proceso intersubjetivo a logrado convertir a las JDV de Sígsig en una evento deportivo de tradición, un lugar estratégico que se manifiesta en su miltidimensionalidad, propiciado el encuentro sociocultural conversacional que enactúa (Massoni, 2003).

Con la emergencia del encuentro sociocultural conversacional, existe un entrecruzamiento de todos los componentes anteriormente descritos y solo puede ser desintegrado de su complejidad para explicarlo, comprenderlo y analizarlo.

Este trabajo se realiza con la finalidad de que se constituya en una alternativa para reactivar las actividades físicas post-pandemia, desde el Ecuador, y para toda "América Latina donde la exclusión (social, política y cultural) es la característica más fuerte y determinante" (Uranga, 2008, p. 4).

\section{Bibliografía}

Alerta. (2014). Informe sobre conflictos, derechos humanos y construcción de paz. Madrid, España. Ediciones Vicens Vives.

Martín Barbero, J. (2003). De los medios a las mediaciones. Bogotá, Colombia. Ediciones Gustavo Gili. S.A. Martínez, J. (2011). El ecologismo de los pobres. Conflictos ambientales y lenguajes de valoración. Barcelona, España. Ediciones Icaria Antrazo.

Massoni, S. (2016a). Avatares del comunicador complejo y fluido. Quito, Ecuador. Ediciones Ciespal.

Massoni, S. (2016b). "Investigación enactiva en comunicación. La participación ciudadana más allá del control en proyectos de comunicación estratégica ambiental” en J. Astudillo y T. Villasante (Comp), Participación ciudadana con metodologías alternativas desde el sur. Cuenca, Ecuador. Ediciones Adya-Yala.

Massoni, S. (2016c). La participación ciudadana es un componente del encuentro sociocultural conversacional (K. Samaniego, Entrevistador).

Massoni, S. (2015). "Comunicación estratégica: Matrices de datos en la Investigación enactiva”, en Revista Latinoamericana de Ciencias de la Comunicación. Año X No18,ALAIC,enero-junio 2013.

Massoni, S. M. (2014). "Plataforma online para el registro y operación de estrategias comunicacionales", en Revista Especializada en Periodismo y Comunicación Vol. 1, No 41, Questión, marzo 2014. Recuperado de: http://perio.unlp.edu.ar/ojs/index.php/question/ article/viewFile/2073/1877.

Massoni, S. (2013a). Metodologías de la comunicación estratégica del inventario al encuentro sociocultural. Rosario, Argentina. Homo Sapiens Ediciones.

Massoni, S. (2013b). "Comunicación estratégica: matrices de datos en la investigación enactiva”, en Revista Latinoamericana de Ciencias de la Comunicación. Año X No 18, ALAIC, enero-junio 2013. Recuperado de: http://rephip.unr.edu.ar/bitstream/handle/2133/5777/ massoni\%20alaic\%2018.pdf?sequence $=7$. 
Massoni, S. (2003). Estrategias de comunicación, un modelo de abordaje de la dimensión comunicacional para el desarrollo sostenible entendido como cambio social conversacional (Tesis doctoral). Universidad de Buenos Aires. Facultad de Filosofía y Letras. Buenos Aires, Argentina.

Massoni, S. (2000). "Estrategias de Comunicación: tiempo de investigarnos vivos", en Revista de Estudios de la Comunicación Social de universidad de Guadalajara, México, No 37, Comunicación y Sociedad, junio 2000. Recuperado de: https://drive.google.com/ file/d/0B9WXVQWpgFVdQmtTYXFiZ1FYOUk/view.

Massoni, S., y Pérez, R. (2009). Hacia una teoría general de la estrategia: El cambio de paradigma en el comportamiento humano, la sociedad y las instituciones. Barcelona, España. Ediciones Ariel.

Maturana, H. y Varela, G. (2002). El árbol del conocimiento. Las bases biológicas del entendimiento humano. Santiago, Chile. Ediciones Universitaria.

Morin, E. (2005). "Epistemología de la complejidad”, en revista del Departamento de Antropología, Geografía e Historia Facultad de Humanidades y Ciencias de la Educación de la Universidad de Jaén. Año X, No 20, Gazeta de Antropología. Recuperado de: http:// www.ugr.es/ pwlac/G20_02Edgar_Morin.html.

Piola, M. (2010). Gestión de conocimiento y comunicación estratégica: su imbricación en el sistema científico tecnológico agropecuario argentino. Un modelo a partir de la deconstrucción/construcción en una Estación Experimental Agropecuaria (Tesis doctoral). Universidad Nacional de Rosario, Facultad de Ciencias Políticas y Relaciones Internancionales. Rosario, Argentina.

Prigogine, I. y. Stengers, I (1997). La nueva alianza. Metamorfosis de la ciencia. Madrid, España. Ediciones Alianza Universitaria.

Samaniego, K. (2017): Comunicación en la Organización Artesanal "Los Macaneros" y su incidencia en las políticas públicas de patrimonio cultural (Tesis Doctoral). Universidad Nacional de la Plata, Facultad de Periodismo y comunicación. La Plata, Ecuador.

Uranga, W. (2016a). La incidencia como camino para la construcción de ciudadanía. Buenos Aires, Argentina. Ediciones Patria Grande.

\begin{abstract}
The world has its sights set on the search for a vaccine to definitively eliminate or neutralize the Corona Virus (Covid-19), declared a pandemic in March 2020. Confinement, social distancing and games on electronic devices are becoming an obstacle to the reactivation of activities in post-pandemic times. It is for this reason that I propose to start with prospective thinking to reduce the negative effects that could affect the moment of reactivating physical activities. With this objective, we will carry out a communicational exploration of the existing intersubjective processes in the Jornadas Deportivas Vacacionales of Sigsig-Ecuador, considered the most constant and oldest in Ecuador, with the intention of examining the communication strategies used in their convocation and participation.
\end{abstract}


Keywords: intersubjectivity - enactive communication - jornadas deportivas vacacionales of sigsig - Ecuador - sociocultural matrices.

Resumo: O mundo está de olho na busca de uma vacina para eliminar ou neutralizar definitivamente o vírus Corona (Covid-19), declarado pandemia em março de 2020. Confinamento, distanciamento social e jogos em aparelhos eletrônicos estão se tornando um obstáculo à reativação das atividades em tempos póst-pandêmicos. É por esta razão que proponho iniciar com o pensamento prospectivo para reduzir os efeitos negativos que podem afetar o momento de reativação das atividades físicas. Com este objetivo, realizaremos uma exploração comunicacional dos processos intersubjetivos existentes nas Jornadas Esportivas de Férias de Sigsig-Equador, consideradas as mais constantes e antigas do Equador, com o intuito de examinar as estratégias de comunicação utilizadas em sua convocação e participação.

Palavras chave: intersubjetividade - comunicação enativa - jornadas deportivas vacacionales de Sigsig - Equador - matrizes socioculturais.

[Las traducciones de los abstracts fueron supervisadas por el autor de cada artículo] 